\title{
An Unusual Presentation of Liver Failure in a Patient with Primary Gastrointestinal Hodgkin's Lymphoma
}

\author{
Gabrielle B. Rocque, ${ }^{1}$ Jeffrey T. Malik, ${ }^{2}$ David T. Yang, ${ }^{3}$ and Julie E. Chang ${ }^{4}$ \\ ${ }^{1}$ Department of Medicine, School of Medicine and Public Health, University of Wisconsin, 600 Highland Avenue, J5/237 CSC, \\ Madison, WI 53792, USA \\ ${ }^{2}$ Department of Pathology, School of Medicine and Public Health, University of Wisconsin, 600 Highland Avenue, \\ Madison, WI 53792, USA \\ ${ }^{3}$ Department of Pathology, School of Medicine and Public Health, University of Wisconsin, 600 Highland Avenue, B4/259 CSC, \\ Madison, WI 53792, USA \\ ${ }^{4}$ Department of Medicine, 4007 Wisconsin Institutes for Medical Research, School of Medicine and Public Health, \\ University of Wisconsin, 1111 Highland Avenue, Madison, WI 53705, USA
}

Correspondence should be addressed to Gabrielle B. Rocque, gblemarbre@hotmail.com

Received 19 July 2010; Revised 15 September 2010; Accepted 5 October 2010

Academic Editor: Jan Delabie

Copyright (C) 2011 Gabrielle B. Rocque et al. This is an open access article distributed under the Creative Commons Attribution License, which permits unrestricted use, distribution, and reproduction in any medium, provided the original work is properly cited.

\begin{abstract}
Introduction. Hodgkin's lymphoma (HL) presenting either with primary bowel involvement or with cholestasis is unusual. The combination of primary gastrointestinal HL presenting with cholestasis and ductopenia has not been previously described. Case Report. We present a case of primary gastrointestinal HL with evidence of liver involvement, but also with prominent ductopenia on liver biopsy and associated intrahepatic cholestasis. A 50-year-old man with a history of Crohn's disease presented with a bowel obstruction, for which he underwent a small bowel resection. Histology revealed HL. His course was complicated by cholestatic liver failure. A subsequent liver biopsy revealed both focal involvement by lymphoma and ductopenia, resembling vanishing bile duct syndrome (VBDS). He was treated with chemotherapy with improvement in his cholestasis, but he eventually succumbed due to further complications of his disease and treatment toxicities. Conclusion. This case of primary gastrointestinal HL associated with ductopenia does not meet classic criteria for VBDS, but the clinical presentation and pathology are suggestive of a VBDS-like paraneoplastic process. Therapies for HL in the setting of cholestatic liver failure require special consideration, but some reports of durable remissions and recovery of liver function have been reported.
\end{abstract}

\section{An Unusual Presentation of Liver Failure in a Patient with Primary Gastrointestinal Hodgkin's Lymphoma}

Hodgkin's lymphoma (HL) presenting with primary bowel involvement is a unique clinical entity that represents less than $5 \%$ of gastrointestinal (GI) lymphomas [1]. More recent criteria for classification of primary GI lymphomas include cases where the bulk of tumor or the dominant disease site involves the GI tract, even in the setting of regional or distant disease sites $[2,3]$.

An equally uncommon clinical presentation of $\mathrm{HL}$ is that of cholestatic liver failure, which may be the result of intrahepatic lymphomatous involvement, extrahepatic compression from mass lesions, or toxic reactions to medications or ionizing radiation. A rare paraneoplastic syndrome previously described in association with HL presenting with cholestatic liver failure is the vanishing bile duct syndrome (VBDS), a subtype of idiopathic cholestasis (IC) [4-6]. Clinical signs of VBDS include intrahepatic cholestasis and evidence of loss of bile ducts (i.e., ductopenia) on biopsy, but without direct involvement of the liver by lymphoma [7]. Outcomes in cases of VBDS and IC range from complete resolution of liver abnormalities with appropriate therapy to permanent hepatic failure and death [5]. There have been multiple case reports of HL-related VBDS and 


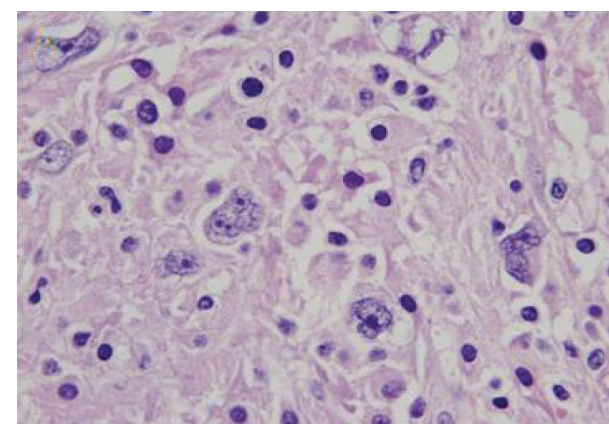

(a)

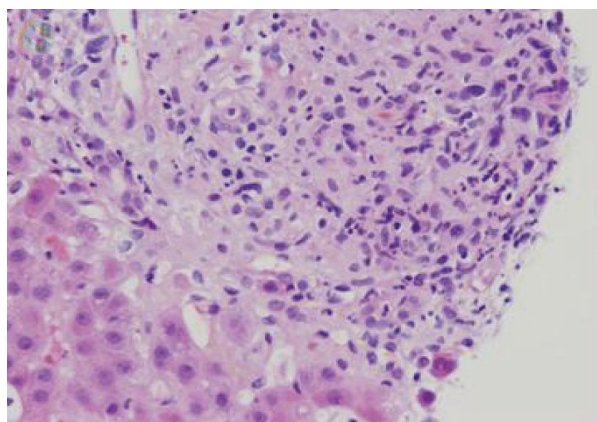

(c)

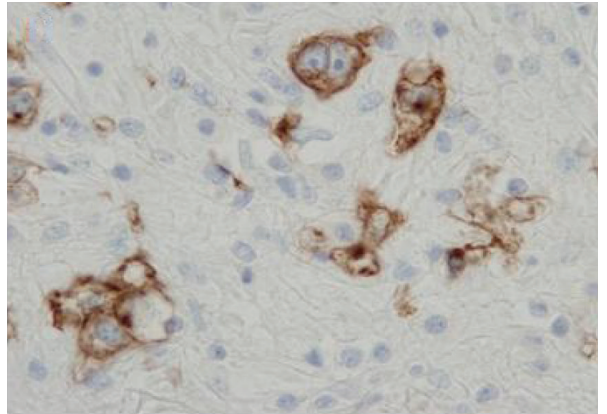

(b)

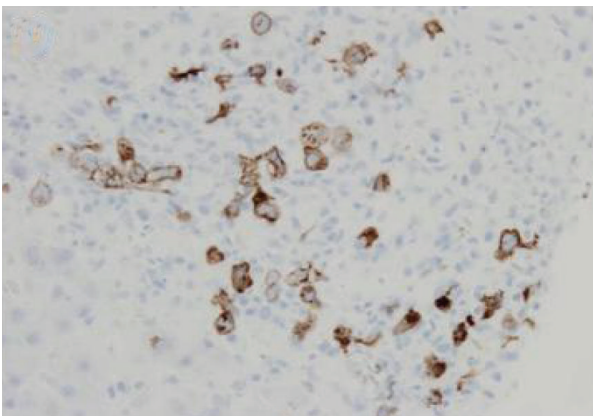

(d)

FIGURE 1: (a) H\&E stain of bowel wall showing large binucleated cells morphologically consistent with Reed-Sternberg cells. (b) CD30 immunostain of bowel wall showing positivity in large binucleated cells. (c) H\&E stain of liver biopsy showing a mixed inflammatory infiltrate among portal tracts with focal obliteration of bile ducts. (d) CD30 immunostain of liver biopsy demonstrating similar positivity in large binucleated cells as seen in the small bowel resection.

IC, all of lymph node origin [5]. We report a case of primary GI HL with evidence of liver involvement, but also with prominent ductopenia on liver biopsy and associated intrahepatic cholestasis. To our knowledge, there has not been a reported case of primary GI HL associated with prominent ductopenia.

A 50-year-old man underwent colonoscopy for evaluation of bloody diarrhea, which demonstrated patchy inflammation of the terminal ileum and colon, suggestive of Crohn's disease. He was treated with sulfasalazine and experienced improvement in his diarrhea. Four years later, he developed worsening abdominal pain, fevers, intermittent bloody diarrhea, anemia, and a 50-pound unintentional weight loss over six months. Computerized tomography (CT) imaging showed small bowel thickening and partial small bowel obstruction. He was treated with steroids and antibiotics with improvement in his symptoms. As his steroids were tapered over several weeks, he developed recurrent abdominal pain and fevers and had an additional 20 -pound unintentional weight loss. Repeat CT imaging revealed marked bowel wall thickening, an enhancing stricture in the distal ileum, and a complex fluid collection within the mesentery communicating with the distal ileum consistent with an abscess. Multiple small adjacent mesenteric lymph nodes $(<1.5 \mathrm{~cm})$ were suspected to be reactive. There was no significant hepatosplenomegaly, and his liver function tests at the time were significant only for an elevated alkaline phosphatase of $964 \mathrm{U} / \mathrm{L}$ (normal 30-130 U/L).

He was started on broad-spectrum antibiotics and underwent a small bowel resection. He continued to have intermittent fevers, despite appropriate antibiotic therapy and a complete infectious workup. He was discharged when he was able to take oral medications but was readmitted the following day with worsening abdominal pain. On physical exam, he appeared jaundiced. Initial laboratory tests were as follows (normal ranges): total bilirubin $10.0 \mathrm{mg} / \mathrm{dL}(\leq 1.4 \mathrm{mg} / \mathrm{dL})$, direct bilirubin $7.8 \mathrm{mg} / \mathrm{dL}$, alkaline phosphatase $447 \mathrm{U} / \mathrm{L}$, AST $137 \mathrm{U} / \mathrm{L}(\leq 50 \mathrm{U} / \mathrm{L})$, ALT $199 \mathrm{U} / \mathrm{L}(\leq 65 \mathrm{U} / \mathrm{L})$, INR 2.0, albumin $1.8 \mathrm{~g} / \mathrm{dL}(3.3-4.7 \mathrm{~g} / \mathrm{dL})$, creatinine $0.7 \mathrm{mg} / \mathrm{dL}(0.6-$ $1.3 \mathrm{mg} / \mathrm{dL})$, and ESR $78 \mathrm{~mm} / \mathrm{hr}(\leq 15 \mathrm{~mm} / \mathrm{hr})$. Hepatitis B, hepatitis C, and HIV serologies were normal. He underwent an emergent exploratory celiotomy, small bowel resection, and repair of an anastomotic leak. Postoperatively, his bilirubin continued to rise to $23.4 \mathrm{mg} / \mathrm{dL}$, and his creatinine increased to $4.8 \mathrm{mg} / \mathrm{dL}$, prompting initiation of hemodialysis.

During the postoperative period following repair of the anastomic leak, the pathology from the initial bowel resection returned showing a transmural chronic inflammatory infiltrate with a background of histiocytes, lymphoctyes, and reactive plasma cells. There were large atypical, binucleated cells noted on histology which stained strongly with CD15, 


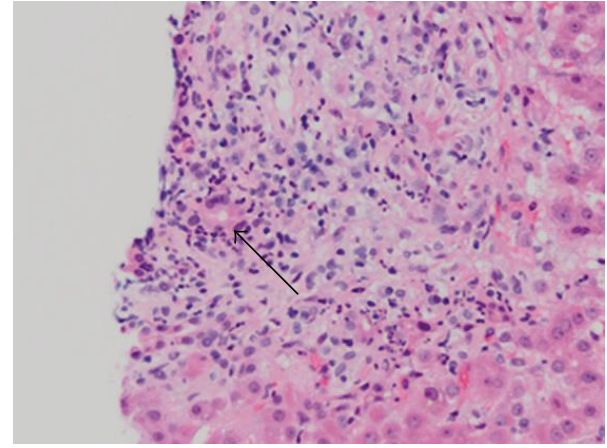

(a)

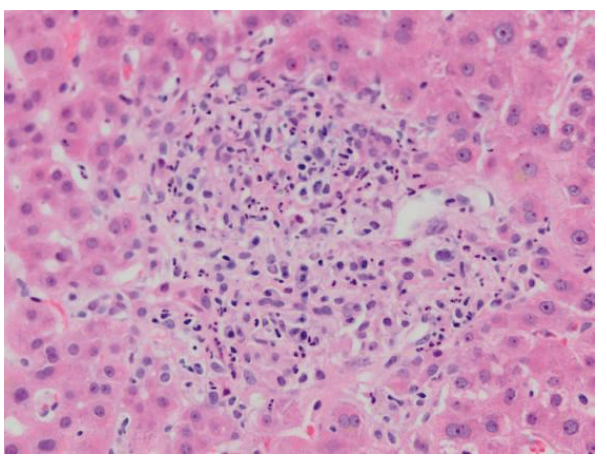

(c)

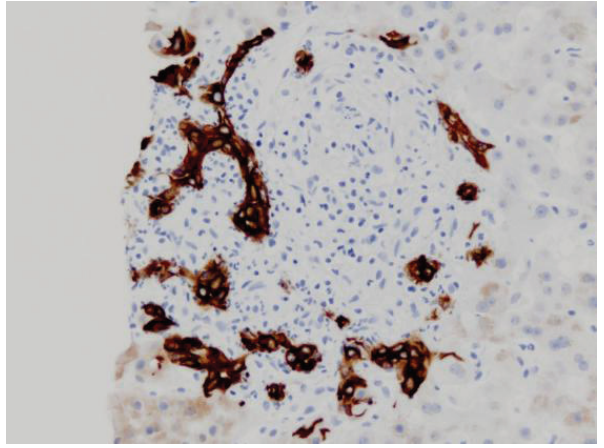

(b)

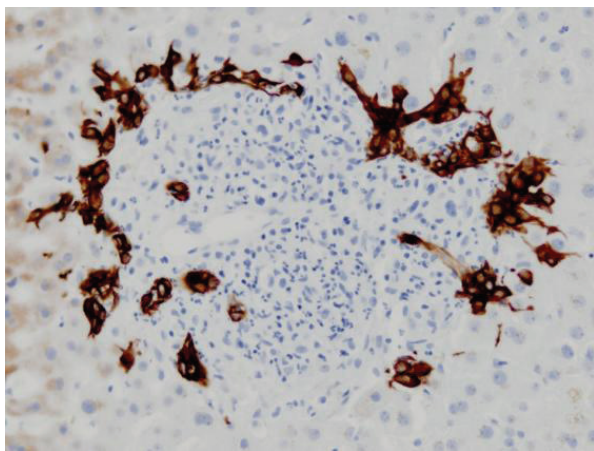

(d)

FIgURE 2: (a) H\&E stain of liver biopsy showing a mixed inflammatory infiltrate within the portal tract comprised of neutrophils, plasma cells, histiocytes, and scattered lymphocytes without definitive Hodgkin's or Reed-Sternberg cells. A damaged bile duct with intraepithelial lymphocytes and periductular neutrophils is shown (arrow). (b) Immunostaining for cytokeratins AE1-3 highlights the damaged bile duct as well as ductular proliferation at the portal tract periphery. (c) A portal tract representative of those assessed as lacking interlobular bile ducts showing the same mixed inflammatory infiltrate as above and no discernable interlobular bile ducts. (d) However, AE1-3 highlights peripheral ductular proliferation in this tract.

CD30, and Epstein Barr virus-encoded RNA (EBER), and were negative for CD45 and CD20, a pattern characteristic of Reed-Sternberg cells in HL (Figures 1(a)-1(b)). The final diagnosis was Epstein-Barr virus- (EBV-) positive classical Hodgkin's lymphoma. A bone marrow biopsy was negative for involvement by lymphoma. A core liver biopsy containing 15 portal tracts showed architecturally intact liver with a mixed inflammatory infiltrate comprised of neutrophils, plasma cells, histiocytes, and scattered lymphocytes and eosinophils within the portal tracts. A few of the portal tracts contained occasional larger cells with atypical lobated nuclei that were morphologically and immunophenotypically similar to the larger binucleated cells seen in the bowel (Figures 1(c)-1(d)), consistent with liver involvement by HL. The liver biopsy also showed evidence of ductopenia, with interlobular ducts present in only eight of the fifteen portal tracts. Most interestingly, there was evidence of ongoing ductal injury within the remaining ducts, with demonstration of intraepithelial lymphocytes, periductular neutrophils, and vacuolated cholangiocytes (Figure 2(a)). While difficult to appreciate by H\&E staining, immunohistochemical staining for cytokeratins AE1-3 highlighted a concomitant proliferation of misshapen ductules around the periphery of the portal tracts (Figures 2(b)-2(d)).
No evidence of biliary ductal obstruction was found by ERCP.

Chemotherapy options for treatment of his HL were limited by his liver failure, renal failure, and poor functional status. He was initially treated with high dose intravenous steroids, with improvement of his liver and renal function. He was transitioned to a modified MOPP regimen, with omission of vincristine and procarbazine due to his renal and liver dysfunction. He received three cycles of a modified MOPP regimen with mechlorethamine and prednisone and had recovery of his kidney function with normalization of his serum creatinine and improvement in his liver function. His total bilirubin improved to as low as $1.9 \mathrm{mg} / \mathrm{dL}$ after 2 cycles of modified MOPP chemotherapy, and his alkaline phosphatase reached a nadir as low as $516 \mathrm{U} / \mathrm{L}$. Unfortunately, his functional status did not improve, and he had multiple further complications including pneumonia, decubitus skin ulcers, severe malnutrition, and steroid myopathy. He was hospitalized with Clostridium difficile colitis during cycle 3 of chemotherapy, with a rapid decline despite appropriate antibiotics and supportive measures; he ultimately died during the course of that hospitalization.

This case involved an atypical presentation of primary bowel involvement by HL with a unique pattern of 
cholestatic liver failure that cannot be entirely attributable to direct liver infiltration by lymphoma. The classic definition of VBDS excludes patients with liver involvement by HL, as VBDS is thought to be due to a paraneoplastic process rather than direct involvement by HL [5]. Patients with cholestatic liver failure related to direct liver involvement by $\mathrm{HL}$ may have some focal portal tract obliteration related to the inflammatory lymphomatous infiltrate but do not demonstrate frank ductopenia [46]. While this case does not meet the criteria for VBDS due to the presence of liver involvement by HL, the pathology suggests that the observed cholestatic liver failure is likely related to both direct lymphomatous invasion of the liver and a potential paraneoplastic process with associated ongoing intrahepatic duct destruction resulting in ductopenia. These observations support the postulate proposed by Liangpunsakul et al. that VBDS is preceded by "early-stage" ductal injury that eventually leads to frank ductopenia [8]. Other hypotheses as to the etiology of ductopenia in VBDS include the presence of cytokines produced by Reed-Sternberg cells that result in hepatotoxicity in select hosts. Such cytokine-mediated cholestasis could result in liver injury through antibody-mediated mechanism or through direct hepatotoxic properties of the cytokine molecule itself [8]. If this theory of cytokine-mediated liver failure is valid, then it would potentially explain the disproportion between the subtle lymphomatous liver infiltration in this patient in relation to the severe degree of cholestatis.

Primary GI HL involving the bowel has been associated with inflammatory bowel disease (IBD) in several case reports [9-12]. Kumar et al. reported 4 cases of GI HL with concurrent EBV infection, with two of the cases occurring in patients with Crohn's disease. DeBenedet et al. described a patient who presented with VBDS and was later diagnosed with HL and IBD. Interestingly, there is one case report of VBDS associated with chronic EBV infection [13]. As EBVpositive HL is relatively common (comprising $20 \%-50 \%$ of HL cases in North America and Europe), [14] it remains unclear if EBV infection is a contributing factor in the rare complication of VBDS.

The treatment strategy for this patient was complicated by his liver failure, renal failure, and poor nutritional status. Chemotherapy with nitrogen mustard agents and steroids may be considered in the setting of liver failure, and there have been successful reports with this approach in HL patients with hepatic dysfunction [11, 15]. This treatment approach was employed for our patient, with marked improvement initially in his liver and renal dysfunction. There are additional data reporting success with radiotherapy in patients with VBDS and HL [5]. However, definitive radiotherapy was not a feasible option in this case of primary GI HL. Ultimately, a primary goal of therapy for HL complicated by VBDS or other IC syndrome is to achieve enough improvement in organ dysfunction as to allow for transition to more definitive therapies (i.e., ABVD chemotherapy regimen) that have reasonably high rates of durable long-term remissions.

\section{Conflict of Interests}

There are no financial or personal relationships between any of the authors and others that might bias their work.

\section{References}

[1] K. S. Crowley, G. Don, G. E. Gibson, C. A. Juttner, and J. R. Miliauskas, "Primary gastrointestinal tract lymphoma. A clinico-pathological study of 28 cases," Australian and New Zealand Journal of Medicine, vol. 12, no. 2, pp. 135-142, 1982.

[2] I. M. Dawson, J. S. Cornes, and B. C. Morson, "Primary malignant lymphoid tumours of the intestinal tract. Report of 37 cases with a study of factors influencing prognosis," British Journal of Surgery, vol. 49, pp. 80-89, 1961.

[3] F. D’Amore, H. Brincker, K. Grønbæk et al., "Non-Hodgkin's lymphoma of the gastrointestinal tract: a population-based analysis of incidence, geographic distribution, clinicopathologic presentation features, and prognosis. Danish Lymphoma Study Group," Journal of Clinical Oncology, vol. 12, no. 8, pp. 1673-1684, 1994.

[4] S. Sherlock, "The syndrome of disappearing intrahepatic bile ducts," Lancet, vol. 2, no. 8557, pp. 493-496, 1987.

[5] A. Ballonoff, B. Kavanagh, R. Nash et al., "Hodgkin lymphoma-related vanishing bile duct syndrome and idiopathic cholestasis: statistical analysis of all published cases and literature review," Acta Oncologica, vol. 47, no. 5, pp. 962-970, 2008.

[6] S. G. Hubscher, M. A. Lumley, and E. Elias, "Vanishing bile duct syndrome: a possible mechanism for intrahepatic cholestasis in Hodgkin's lymphoma," Hepatology, vol. 17, no. 1, pp. 70-77, 1993.

[7] B. A. Bouroncle, J. W. Old Jr., and A. G. Vazques, "Pathogenesis of jaundice in Hodgkin's disease," Archives of Internal Medicine, vol. 110, pp. 872-883, 1962.

[8] S. Liangpunsakul, P. Kwo, and G. K. Koukoulis, "Hodgkin's disease presenting as cholestatic hepatitis with prominent ductal injury," European Journal of Gastroenterology and Hepatology, vol. 14, no. 3, pp. 323-327, 2002.

[9] M. Vanbockrijck, M. Cabooter, J. Casselman, J. Vanvuchelen, A. Van Hoof, and P. Michielssen, "Primary Hodgkin disease of the ileum complicating Crohn disease," Cancer, vol. 72, no. 5, pp. 1784-1789, 1993.

[10] S. Kumar, F. Fend, L. Quintanilla-Martinez et al., "EpsteinBarr virus-positive primary gastrointestinal Hodgkin's disease: association with inflammatory bowel disease and immunosuppression," American Journal of Surgical Pathology, vol. 24, no. 1, pp. 66-73, 2000.

[11] A. T. DeBenedet, C. L. Berg, K. B. Enfield, R. L. Woodford, A. K. Bennett, and P. G. Northup, "A case of vanishing bile duct syndrome and IBD secondary to Hodgkin's lymphoma," Nature Clinical Practice Gastroenterology and Hepatology, vol. 5, no. 1, pp. 49-53, 2008.

[12] B. Vincenzi, E. Finolezzi, C. Fossati et al., "Unusual presentation of Hodgkin's disease mimicking inflammatory bowel disease," Leukemia and Lymphoma, vol. 42, no. 3, pp. 521-526, 2001.

[13] K. Kikuchi, H. Miyakawa, K. Abe et al., "Vanishing bile duct syndrome associated with chronic EBV infection," Digestive Diseases and Sciences, vol. 45, no. 1, pp. 160-165, 2000. 
[14] K. J. Flavell and P. G. Murray, "Hodgkin's disease and the Epstein-Barr virus," Molecular Pathology, vol. 53, no. 5, pp. 262-269, 2000.

[15] I. M. Ghobrial, R. C. Wolf, D. L. Pereira et al., "Therapeutic options in patients with lymphoma and severe liver dysfunction," Mayo Clinic Proceedings, vol. 79, no. 2, pp. 169-175, 2004. 


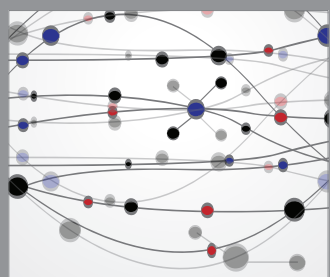

The Scientific World Journal
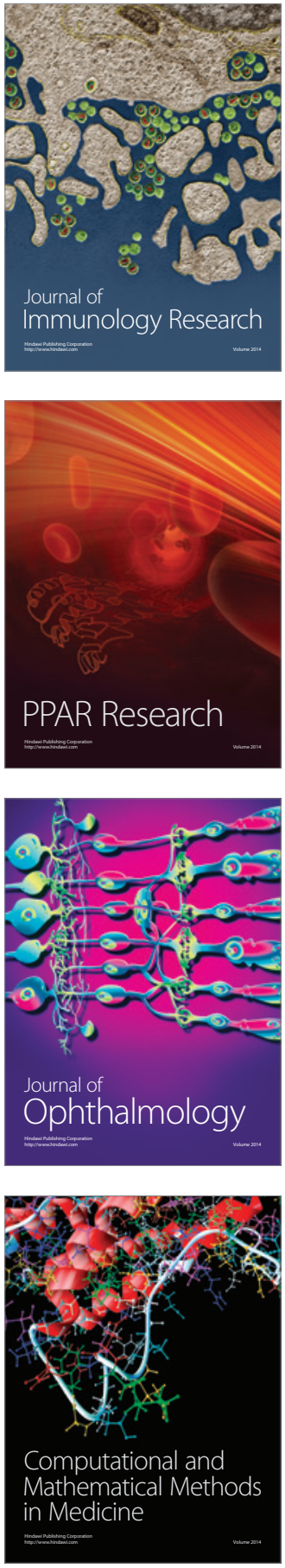

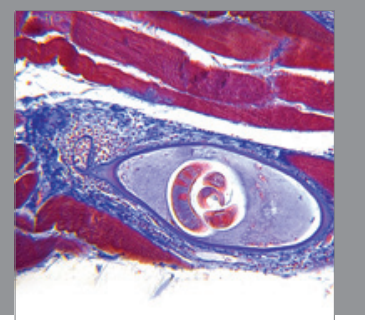

Gastroenterology

Research and Practice
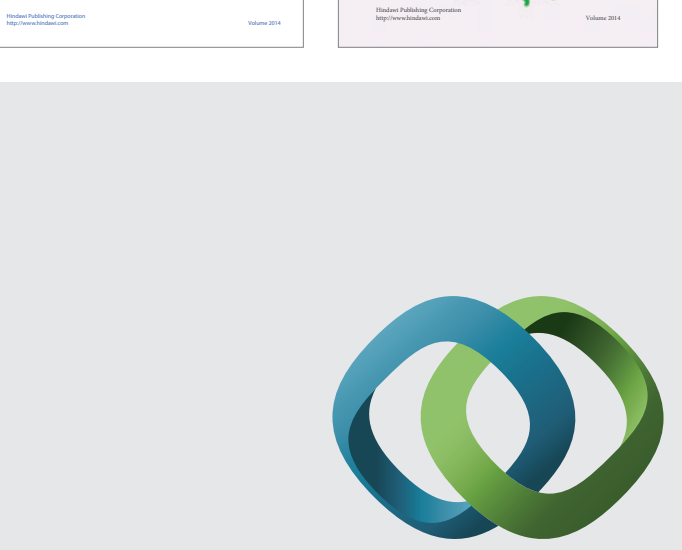

\section{Hindawi}

Submit your manuscripts at

http://www.hindawi.com
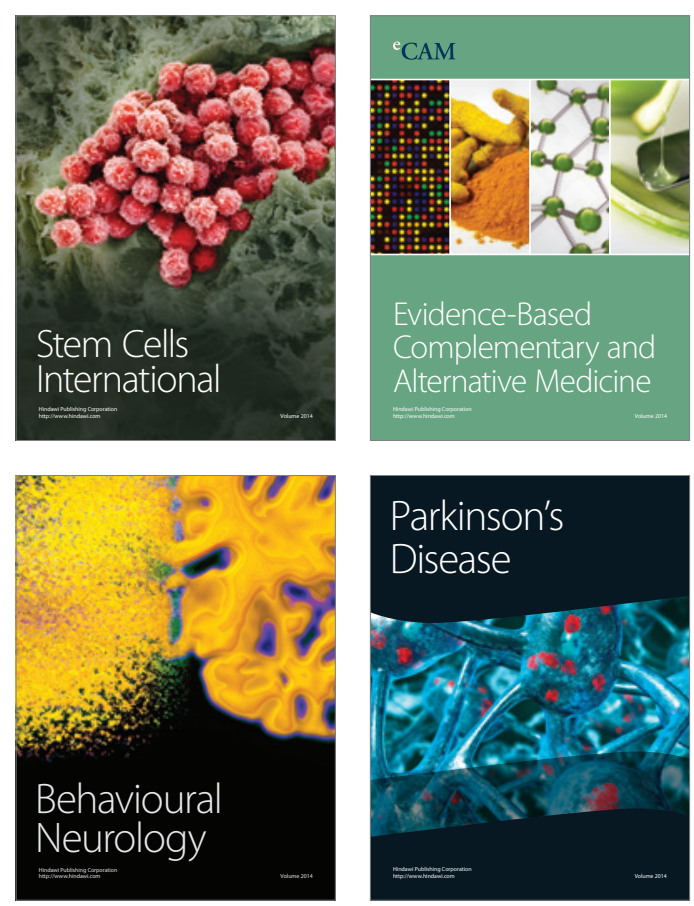

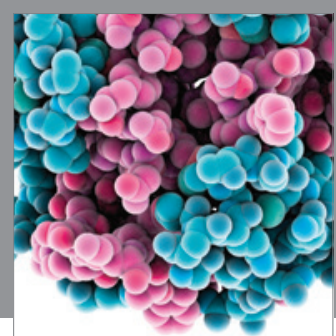

Journal of
Diabetes Research

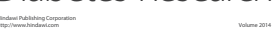

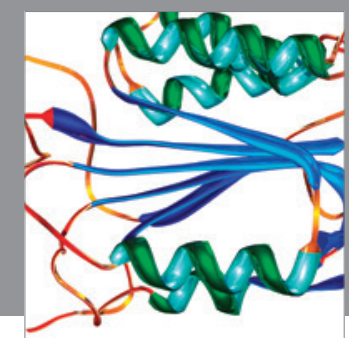

Disease Markers
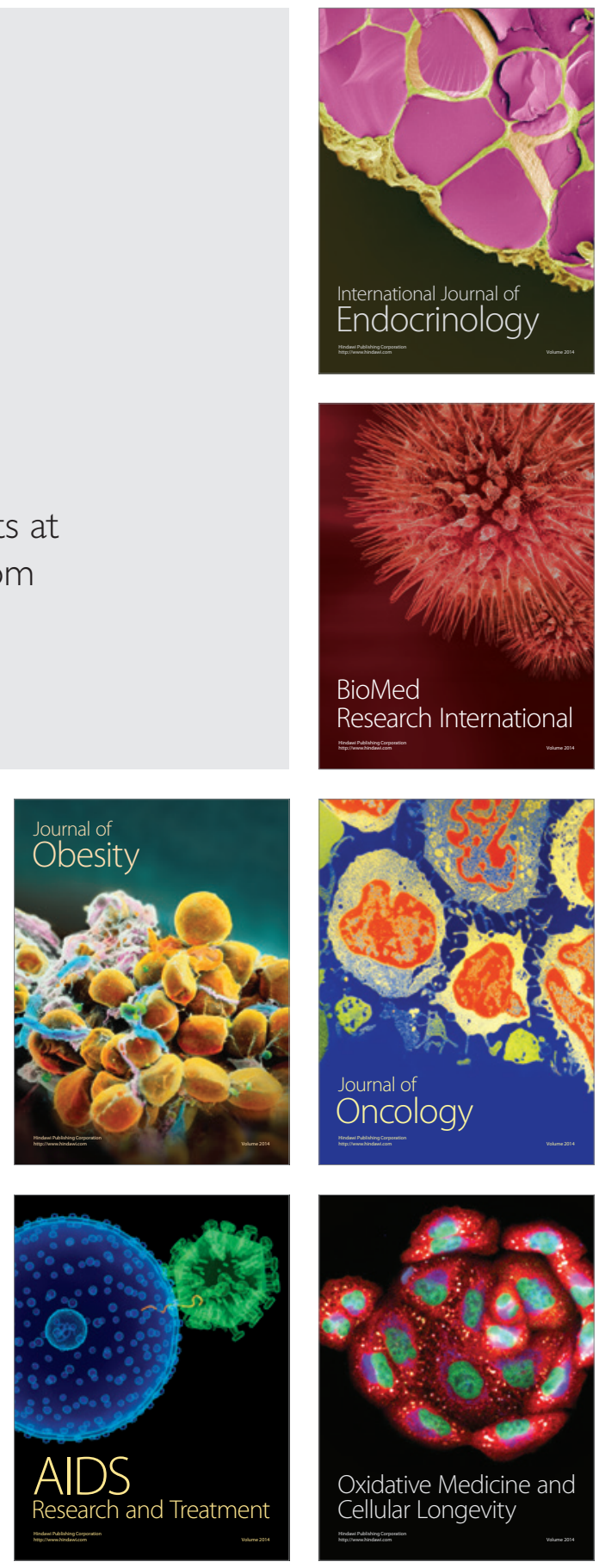